\title{
Novelty Au Nanoparticles with Different Nano Sizes as an Acidity Sensor
}

\author{
Zehraa Najim Abdul-Ameer \\ Department of Remote Sensing and GIS, College of Science, University of Baghdad, Baghdad 10001, Iraq
}

Corresponding Author Email: zehraanajim@gmail.com

https://doi.org/10.18280/rcma.310308

Received: 1 March 2021

Accepted: 30 April 2021

\section{Keywords:}

gold, nanoparticles, size, acidity, sensor, reagent, Turkuvich method

\begin{abstract}
The amazing wide range, applications of gold nanoparticles corresponding to excellent optical and electronic properties enhance utilization of Au NP as PH sensor. Gold nanoparticles were synthesized in simple and rapid Turkevich method to accomplish optical sensing technologies covalent or non-covalent, bonds formation causes, aggregation of the nanoparticles in the target substance. Au nanoparticles characterization was studied using XRD, UV-spectroscopy and SEM. XRD indicate polycrystalline. Nanoparticles with two different sizes according to reducing agent amount to obtain $2030 \mathrm{~nm}$. Optical energy gap was calculated for both particle size to find it (2.13) eV for $30 \mathrm{~nm}$ particle size and (2.33) eV for 20nm.Scanning Electron Microscope showed spherical shape particles. PH test were performed to investigate efficiency of Au Np's for PH sensor and that is found to be excellent results for both sizes with more efficiency for $30 \mathrm{~nm}$ due to its rough surface. Which is a fantastic tool and measurement device to test the $\mathrm{pH}$ of soil, food, and other puncture-worthy applications.
\end{abstract}

\section{INTRODUCTION}

Gold nanoparticles are widely used in many fields as preferred materials for their unique optical and physical properties, such as surface Plasmon oscillations for labeling, imaging, and sensing. The amazing, optical, properties of metal especially for interaction with the electromagnetic, visible light, this is due to its good resistance against the corrosion. These properties qualify $\mathrm{Au}$ nanoparticles to be utilized as PH sensor which is a fantastic [1, 2]. Gold nanoparticles attracted attention, due to its special, properties: the surface, Plasmon, resonance (SPR); and surface to volume ratio, besides high Surface Energy, quantum confinement and quantum dot $[3,4]$.

Abdulghani and Hussain [5] studied preparation of $\mathrm{Au}$ nanoparticles using Turkevich Method. Niu et al. studied the influence of citrate and Au concentration, reaction temperature and time, are parameters, to control, shape [6]. Sau et al. used the water phase oxidation-reduction method known as seed growth method to prepare small gold nanoparticles as seeds. Gold nano Particles can be synthesized with different sizes and, morphologies with spherical rod and triangular shape controlled by the crystal ratio to growth solution $[7,8]$. Turkevich method for AuNPs synthesis was utilized for fabrication of $\mathrm{SiO}_{2} @ \mathrm{Au}$ and $\mathrm{TiO}_{2} @ \mathrm{Au}$ nanostructures. The nanostructures synthesized using this method were compared to $\mathrm{SiO}_{2} @ \mathrm{Au}$ and $\mathrm{TiO}_{2} @ \mathrm{Au}$ nanostructures obtained by deposition of Turkevich AuNPs on amino-functionalized SiO2 and $\mathrm{TiO}_{2}$ corese significant red shift and broadening of the spectrum in the case of AuNPs decorated $\mathrm{TiO}_{2}$ cores are likely related to high refractive index of $\mathrm{TiO}_{2}$ and dense packing of AuNPs on $\mathrm{TiO}_{2}$ particles surface, resulting in strong AuNPs plasmon-plasmon coupling as studied by Yahya [9].

\section{EXPERIMENTAL METHOD}

Turkevich Method was used to synthesize $\mathrm{Au}$ Nano particles solution. $1 \mathrm{~g}$ of Chloroauric acid was dissolved in $50 \mathrm{ml}$ of deionized water with $58.86 \mathrm{mM}$ molarity (Riedel-De Haen Ag / Seelz- Hannover, Germany) then withdraw $150 \mu 1$ of solute on the heat it up to $100^{\circ} \mathrm{C}$ to add $500 \mu$ Trisodium Citrate Dihydrate solution of $34 \mathrm{Mm}$ (Panreac AppliChem / Bercelona, Spain) at once with continuous stirring. We will notice changes in the color of solution: yellow-clear whitelight gray-dark gray-dark violet-red-wine, until it becomes red-wine, immediately stop heating and stirring and leave the solution to get cool.

\section{TURKEVICH METHOD}

One of the very well-known procedures for AuNPs synthesis is based on the diminishment of $\mathrm{HAuCl} 4$ by citrate in water, which was to begin with planned by Turkevich in 1951 as shown in Figure 1. In this strategy, the HAuCl4 arrangement is bubbled, and the trisodium citrate dihydrate is at that point quickly included into the bubbling arrangement under vigorous stirring. After a couple of minutes, the color of the arrangement changes from light yellow to wine ruddy. This strategy comes about in AuNPs measuring approximately 20 $\mathrm{nm}$ in breadth. In this procedure, citrate ions play a double part, as both stabilizing and diminishing specialists (Turkevich et al. 1951, Yahya 2017) [8, 9].

Puntes et al. have studied that a process with inverse sequence as addition of reagents (addition of HAuCl4 into a boiling sodium citrate solution) leads to the production of AuNPs with small size and a narrow size distribution as shown in Figure $2[9,10]$. 

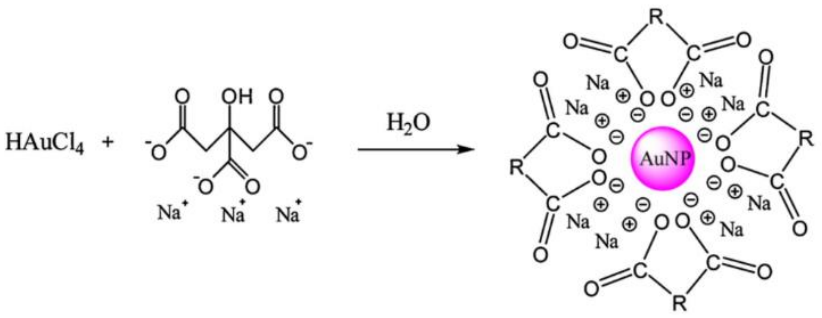

$$
\mathrm{R}=\prod_{\mathrm{CH}_{2}-}^{\mathrm{CH}_{2}-}
$$

Figure 1. AuNP ion utilizing the Turkevich method in gold nanoparticle synthesis [10]

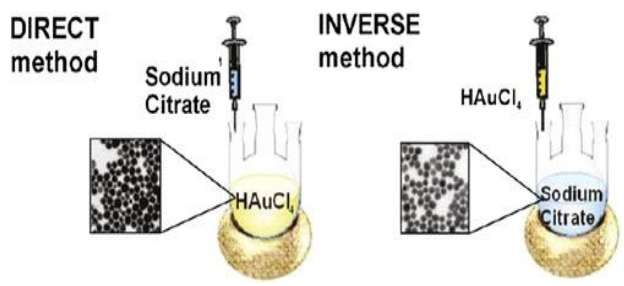

Figure 2. Direct and inverse sequence of reagent addition

\section{CHARACTERIZATION OF Au NANOPARTICLES}

\subsection{XRD characteristics}

Crystallographic information was analyzed from XRD to study structure and morphology using Shimadzu instrument, X-ray tube with target, Cuk $\alpha$ radiation, voltage, $40.0(\mathrm{kV})$, current $30.0(\mathrm{~mA})$ to calculate, grain, size average (D) was determined using Scherer's equation [11, 12]:

$$
\mathrm{D}=0.94 \lambda / \beta \operatorname{COS} \Theta
$$

where, $(\lambda=1.542, \AA)(\mathrm{CuK \alpha}), \beta$ is the full width at half, maximum, of the line (FWHM) and $2 \theta$ scanning range was $(30-80)$ as, in Table 1. Figure 3 shows XRD structures and for $\mathrm{Au}$ nanoparticles. From The pattern we can notice, that preferential, crystal at $\Theta=38.1^{\circ}, 44.3^{\circ}, 64.5^{\circ}$, and $77.7^{\circ}$, corresponding, to (111), (200), (220), (311) characteristic peaks (Figure 3). Scherer's equation is used to determine average grain size $(20,30) \mathrm{nm}$.

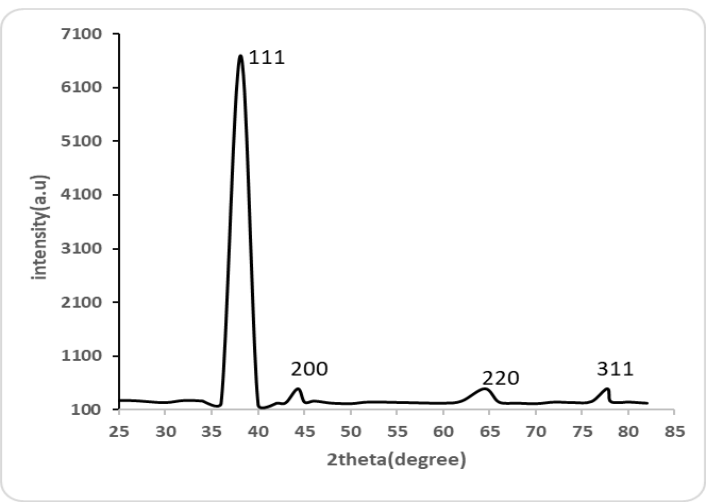

Figure 3. XRD characteristic for Au nanoparticles

\subsection{Optical properties}

Shimadzu, Spectrum, of, Au, nanoparticles, of, range 190$1100 \mathrm{~nm}$ as shown in Figure 4, to study the optical, properties. Photoluminescence, at room, temperature was measured, using, spectrophotometer of Elico with range (300-900) nm wavelength, range. It was noticed that Energy gap values, have small difference due to agglomeration of these particles that as in Table 1. While Photoluminescence, were tested for, the, samples, using excitation wavelength of $320 \mathrm{~nm}$. the spectrum. Figure 5 showed sharp peak for Au nanoparticles $20 \mathrm{~nm}$ with peak centered at $523 \mathrm{~nm}$. Wide and broad peak noticed at ( $\mathrm{Au}$ $30 \mathrm{~nm}$ ) with SPR band centered at $560 \mathrm{~nm}$. it is found that gold nanoparticles can be synthesized in a wide range of sizes, from 20 to $120 \mathrm{~nm}$, with different characterization, taking in to account the previous work of Turkevich et al. [8], which results in numerous steps of essential and multiple steps of primary and secondary clustering leading to polycrystallites [13].

Table 1. Structural and optical properties of Au nano, particles with two different sizes

\begin{tabular}{cccccc}
\hline & $\begin{array}{c}\mathbf{E g} \\
(\mathbf{e V})\end{array}$ & $\begin{array}{c}\mathbf{D} \\
(\mathbf{n m})\end{array}$ & $\begin{array}{c}\text { B } \\
(\mathbf{F W H M}) \\
(\mathbf{r a d})\end{array}$ & $\boldsymbol{\Delta}$ & $\boldsymbol{\varepsilon}$ \\
\hline $\begin{array}{c}\mathrm{Au} \\
(30 \mathrm{~nm})\end{array}$ & 2.13 & 22 & 0.32 & 0.002 & 188 \\
$(20 \mathrm{~nm})$ & 2.33 & 6 & 0.87 & 0.027 & 162 \\
\hline
\end{tabular}

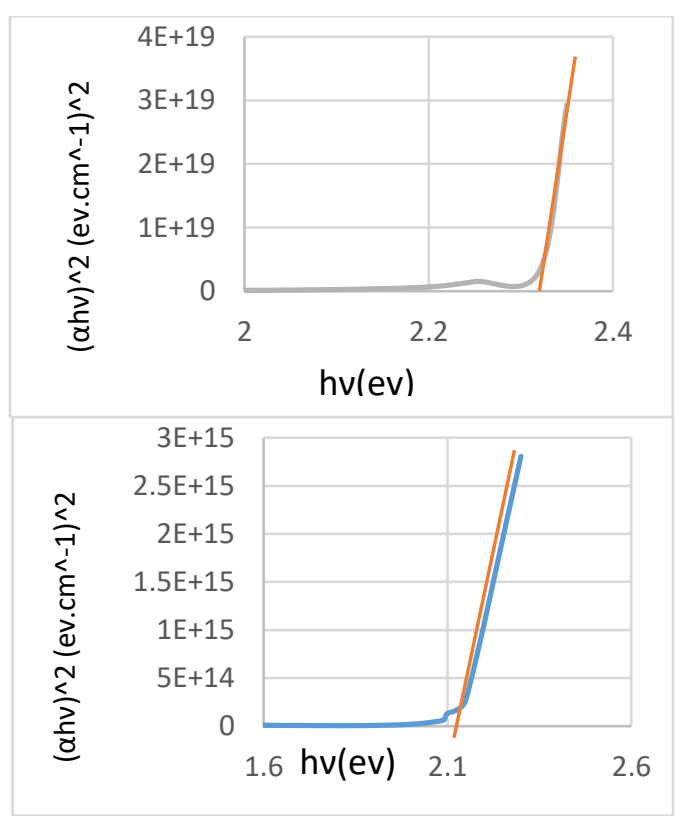

Figure 4. Optical properties of a-Au $(20 \mathrm{~nm}) \mathrm{b}-\mathrm{Au}(30 \mathrm{~nm})$

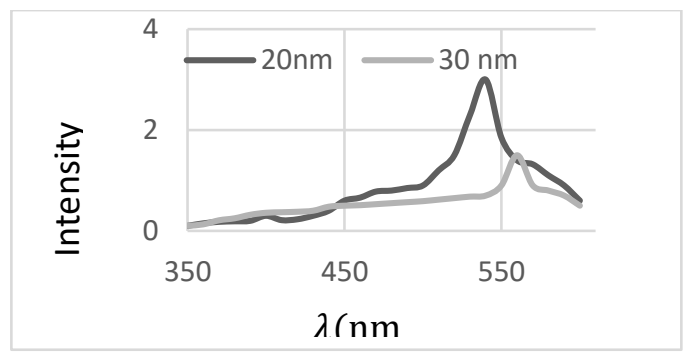

Figure 5. Photoluminescence intensity as function of wavelength 
Maximum absorption wavelength at $523 \mathrm{~nm}$ was for $20 \mathrm{~nm}$ gold, nanoparticles, wine red color, the maximum adsorption, wavelength and, change in color (faint yellow, wine red, purple red, blue) with increase in, the particle size $(30 \mathrm{~nm})$ in the inter particle distance causes a red shift $[14,15]$.

\subsection{Tunnelling Electron Microscope (TEM)}

Figure 6 shows a HR Tunnelling Electron Microscope image for gold nanoparticles to measure the size of the particles. TEM images showed spherical shape gold nanoparticles and this agree with Zhao et al. [10]. TEM images of $(20 \mathrm{~nm}) \mathrm{Au}$ Nps with $500 \mu \mathrm{l}$ Trisodium Citrate Dihydrate solution. $30 \mathrm{~nm}$ images showed more agglomeration with 300 $\mu 1$ Trisodium Citrate Dihydrate causing larger particle size with moderate roughness.
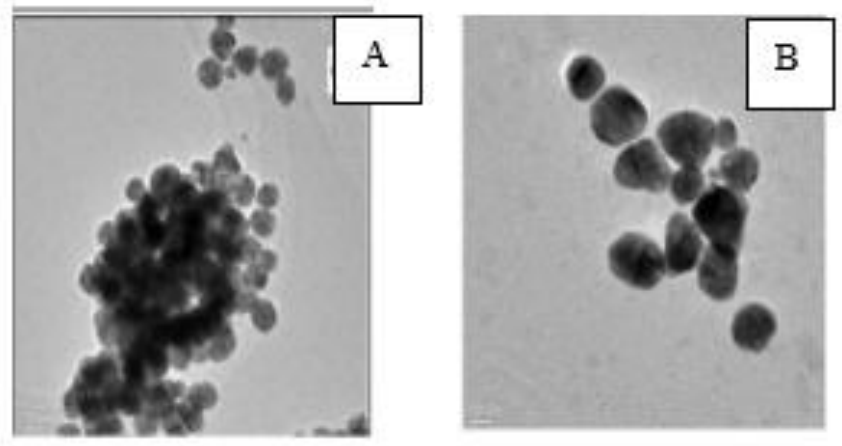

Figure 6. Tunneling electron microscope: a-20 nm b- $30 \mathrm{~nm}$

\subsection{Acidity test procedure}

Acidity measurement include several steps: setting the, $\mathrm{PH} / \mathrm{mV}$ switch on the $\mathrm{PH}$ meter to the $\mathrm{mV}$ position then rinsing the electrode with (DI) water after that insert the electrode in the $(\mathrm{PH}=7)$ buffer for 30 minutes then record the polarity and the $\mathrm{mV}$ reading repeat the same steps for $4 \mathrm{PH}$ buffer and the difference between the two $\mathrm{mV}$ readings was determined which, is the electrode span then divide the electrode's span by the theoretical span of $176.9 \mathrm{mV}$ (at $250^{\circ} \mathrm{C}$ ), then multiply by 100 and determine the slope of the electrode (Figure 7).

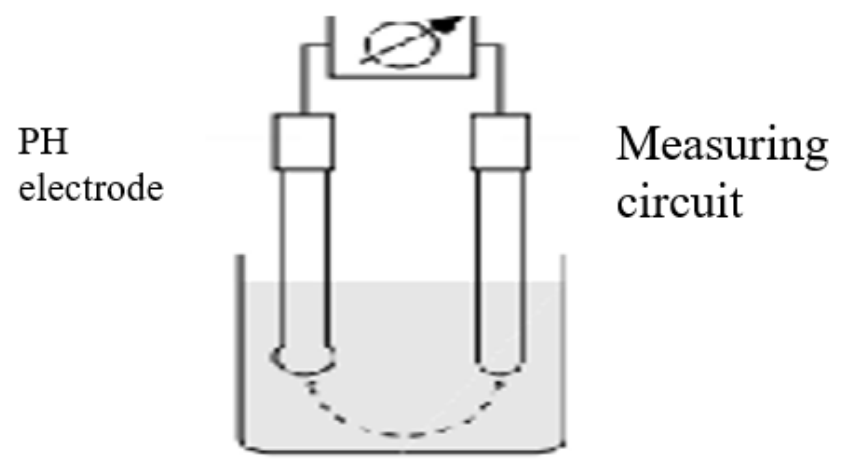

Figure 7. Measuring circuit

Figure 8 shows the acidity properties for both gold particle sizes. it is obvious from the figure that the Au np with $30 \mathrm{~nm}$ has better sensing properties [14, 15].

Recently, the chemicals internals of the sensors and the junctions that separate them from the external world come in a few variations material differences; but for the sake of application most sensors utilize silver chloride or potassium chloride and some types of ceramic junction. Increasing the robustness of these materials, or adding more junctions, would again be for more harsh applications and contaminated environments such as gold nanoparticles which is ideal with zero residues in the environment [16-18].

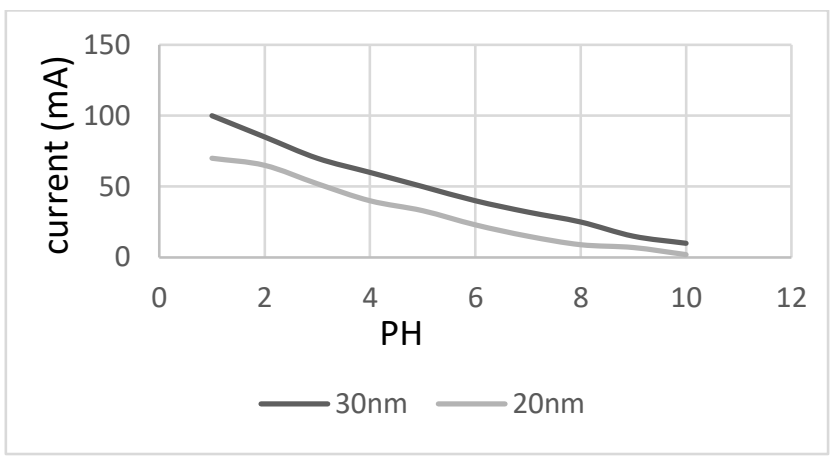

Figure 8. Current as function of PH: $20 \mathrm{~nm}, 30 \mathrm{~nm}$

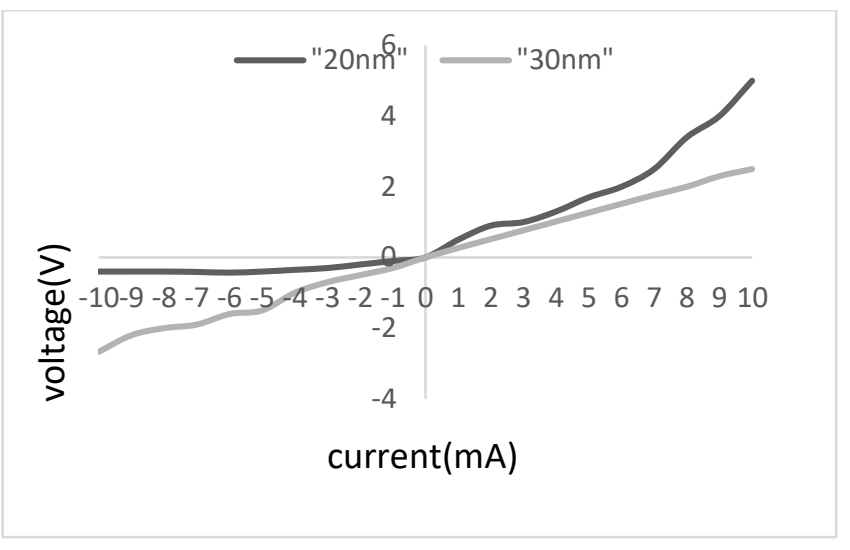

Figure 9. I-v characteristic properties of Au nanoparticles

One of the indications of efficiency of any sensor is the I-V characteristics as shown in Figure 9.

\section{RESULTS AND DISCUSSION}

Abdul-Ameer [13] research discussed different reasons for emission obtained from $\mathrm{Au}$, nanoparticles of size $18 \mathrm{~nm}$, and $13 \mathrm{~nm}$. With (PLE) maximum occurs at approximately $255 \mathrm{~nm}$ which is about half the SPR peak position of $526 \mathrm{~nm}$ which is approximately close to our study. The optical properties of such materials depend on chemistry of their surfaces and on controlling of the dimensions of the crystal. Surface Plasmon (SP) is responsible in the colour of nanomaterial. As the volume, ratio increase the surface. Plasmon resonance, shift to the right or red shift occurred, the results agree with those of Tapan, K. Sau et al. [7] and become more broadening which indicates a different size or the size of $\mathrm{Au}$, NPs increased while the size of $\mathrm{Au}$, NPs become mono disperse when the volume ratio decreased, agree with the research results of Eustis and El-Sayed [18] and Shah et al. [19].

This research studied preparation of $\mathrm{PH}$ sensor which is found to have different sizes of $\mathrm{Au} \mathrm{Nps}(20,30 \mathrm{~nm})$ with excellent optical properties for $20 \mathrm{~nm}$ in accordance to Au Nps with $30 \mathrm{~nm}$ in spite of that it was found that Au, Nps with 30 $\mathrm{nm}$ particle size have good sensing, properties due to roughness. 


\section{CONCLUSIONS}

Gold nanoparticles were, synthesized in simple and rapid Turkevich method. Formation of covalent, or non-, covalent. Bonds with, the target, substance induce special optical sensing, technologies. characteristics indicate poly crystalline nanoparticles with two different sizes according to reducing agent amount to obtain 20,30 nm with XRD. optical energy gap was calculated for both particle size to find it (2.13) $\mathrm{eV}$ for $30 \mathrm{~nm}$ particle size and (2.33) eV for $20 \mathrm{~nm}$. Scanning Electron Microscope showed spherical shape particles .PH test were performed to investigate efficiency of $\mathrm{Au} \mathrm{Nps}$ for $\mathrm{PH}$ sensor and that is found to be excellent results for both sizes with more efficiency for $30 \mathrm{~nm}$ due to its rough surface.

We conclude that we have, succeeded in synthesizing of $\mathrm{Au}$, NPs, on the surface of, Psi using a cost-effective, and simple, method of Turkevich. The current density was calculated, of the deposition process to indicate, the size of the, formed $\mathrm{Au}$ NPs. These properties qualify Au nanoparticles to be utilized as $\mathrm{PH}$ sensor which is a fantastic tool and measurement device to test the $\mathrm{pH}$ of soil, food, and other puncture-worthy applications which is recorded for Au nanoparticles with 30 $\mathrm{nm}$ size due to agglomeration and increased roughness which is important for sensing process.

\section{ACKNOWLEDGEMENTS}

Ministry of science and technology and also laser institute for higher studies.

\section{REFERENCES}

[1] Nagarian, R., Hatton, T.A. (2008). Nanpparticles: Synthesis, Stabilization, Passivation, and Functionalization Series. American Chemical Society, Washingtone.

[2] Schmid, G., Corian, B. (2003). Nanoparticulated gold: Synthesis, structures, electronics, and reactivities. Eur. J. of Inorg, Chem., 2003(17): 3081-3098. https://doi.org/10.1002/ejic.200300187

[3] Turkevich, J., Stevenson, P.C., Hillier, J. (1951). A study of the nucleation and growth processes in the synthesis of colloidal gold". Discuss. Faraday. Soc., 11: 55-75. https://doi.org/10.1039/DF9511100055

[4] Kimling, J., Maier, M., Okenve,B., Kotaidis, V., Ballot, H., Plech, A. (2006). Turkevich method for gold nanoparticle synthesis revisited. J. Phys. Chem. B., 110(32): https://doi.org/10.1021/jp061667w

15700-15707.

[5] Abdulghani, A., Hussain, R,K. (2014). Synthesis of gold nanoparticles via chemical reduction of $\mathrm{Au}$ (III) Ions by Isatin in aqueous solutions: Ligand concentrations and pH effect. Baghdad Science Journal, 11(3): 1201-1216.

[6] Niu, W.X., Zheng, S.L., Wang, D.W., Li, X.Q., Han, S., Chen, J., Tang, Z.Y., Xu, G.B. (2009). Selective synthesis of single-crystalline rhombic dodecahedral, octahedral, and cubic gold nanocrystals. J. Am. Chem. Soc, 131(2): 697-703. https://doi.org/10.1021/ja804115r

[7] Sau, T.K., Murph, C.J. (2004). Room temperature, highyield synthesis of multiple shapes of gold nanoparticles in aqueous solution. J. Am. Chem. Soc., 126(28): 86488649. https://doi.org/10.1021/ja047846d
[8] Turkevich, J., Stevenson, P.C., Hillier J. (1951). Nucleation and growth process in the synthesis of colloidal gold. Discuss Faraday Soc., 11: 55-75.

[9] Yahya, Z. (2017). Plasmonic hot gold nanoparticles for treatment of cancer cell line. $\mathrm{PhD}$ thesis, Baghdad University.

[10] Zhao, P.X., Li, N., Astruc, D. (2013). State of the art in gold nanoparticle synthesis. Coordination Chemistry Reviews, 257(3-4): 638-665. https://doi.org/10.1016/j.ccr.2012.09.002

[11] Chugh, H., Sood, D., Chandra, I., Tomar, V., Dhawan G., Chandra, R. (2018). Role of gold and silver nanoparticles in cancer nano-medicine. Artificial Cells, Nanomedicine, and Biotechnology, 46: 1210-1220. https://doi.org/10.1080/21691401.2018.1449118

[12] Abdul-Ameer, Z.N., Agool, I.R. (2015). Structural and optical properties of $\mathrm{ZnO}$ electrodeposition method. Advances in Environmental Biology, 9(24): 445-449.

[13] Abdul-Ameer Z.N. (2016). Effect of concentration on characterization of $\mathrm{MgO}$ nanoparticles using chemical bath method. Advances in Natural and Applied Sciences Journal, 10(12): 1998-1090.

[14] Anwar, A., Minhaz, A., Khan, N.A. , Kalantari, K. Afifi, A.B.M., Shah, M.R. (2018). Synthesis of gold nanoparticles stabilized by a pyrazinium thioacetate ligand: A new colorimetric nanosensor for detection of heavy metal Pd(II). Sens. Actuators B-Chem., 257: 875881. https://doi.org/10.1016/j.snb.2017.11.040

[15] Dong, H., Zou, F., Hu, X., Zhu, H., Koh, K., Chen, H. (2018). Analyte induced AuNPs aggregation enhanced surface plasmon resonance for sensitive detection of paraquat. Biosens. Bioelectron., 117: 605-612. https://doi.org/10.1016/j.bios.2018.06.057

[16] Zhao, J.Y., Friedrich, B. (2015). Synthesis of gold nano particles via chemical reduction methods. NANOCON 2015. https://doi.org/10.13140/RG.2.2.28933.35049

[17] Lin, H.Y., Chen, Y.F. (2005). Enhanced luminescence by second-harmonic surface plasmon resonance. Proc. Emerging Information Technology Conference, pp. 7803-9328. https://doi.org/10.1109/EITC.2005.1544349

[18] Eustis, S., El-Sayed, M.A. (2006). Why gold nanoparticles are more precious than pretty gold Noble metal surface plasmon resonance and its enhancement of the radiative and nonradiative properties of nanocrystals of different shapes. Chem. Soc. Rev., 35: 209-217. https://doi.org/10.1039/B514191E

[19] Shah, M., Badwaik,V., Kherde, Y. et al. (2014). Gold nanoparticles: Various methods of synthesis and antibacterial applications. Frontiers in Bioscience, 19(8): 1320-1344. https://doi.org/10.2741/4284

\section{NOMENCLATURE}

$\begin{array}{ll}\mathrm{E}_{\mathrm{g}} & \text { Enrgy band gap }(\mathrm{eV}) \\ \mathrm{D} & \text { Crystallite size }(\mathrm{nm}) \\ \mathrm{V} & \text { Voltage }(\mathrm{V}) \\ \mathrm{I} & \text { Current }(\mathrm{A})\end{array}$

\section{Greek symbols}

Full width at half maximum(rad) Micro strain 
Absorption coefficient

Wavelength $(\mathrm{nm})$

Diffraction angle(deg)

Frequency(hz)

\section{Subscripts}

$\mathrm{Au}$ np

XRD

PL

$\mathrm{PH}$

SPR

HR

TEM nanoparticle

Xray diffraction

Photoluminescence

acidity

surface Plasmon Resonance

high resolution

tunneling electron microscope 